\title{
Human Fundamental Upward Movement; Spiral Trajectory and Walking of 3D + 1D Space and Time
}

\author{
Hiroko Tadaura $^{1^{*}}$ \\ ${ }^{1}$ Graduate School of Health and Welfare, International University of Health and Welfare, Tokyo, Japan
}

Received Date: December 15, 2020 Accepted Date: January 18, 2021 Published Date: March 30, 2021

Citation: Hiroko Tadaura (2021). Human Fundamental Upward Movement; Spiral Trajectory and Walking of 3D + 1D Space and Time. POJ NursPrac Res. 5(1):1-7. DOI: https://doi.org/10.32648/2577-9516/5/1/3.

Corresponding Author: Prof. Hiroko Tadaura, PhD, Graduate School of Health and Welfare, International University of Health and Welfare, 4-1-26 Akasaka, Minato-ku, Tokyo, 107-8402, Japan; Email: hiroko@tadaura.com.

\begin{abstract}
Background: There are few biomechanical studies on the physical transfer of a person lying on a floor or bed perpendicular to the direction of gravity. Basic biomechanics research can be an important source. This study aimed to analyze the biomechanical properties of the upward movement of the floor in the supine position.
\end{abstract}

Methods: Healthy volunteers were recruited by snowball sampling. The movement from the supine position to upward movement on the carpet floor was repeated three times. The three procedures were analyzed with an 11 segments model using a 3D motion analysis Move Tr 3D (Library@) under the 4 CCD cameras. The analysis of the motion trace was examined. The movement of the reflection marker with respect to the Z-axis was analyzed with the vertical direction of the gravity as the Z-axis. It was observed from the XY plane, XZ plane, ZY plane, and how many dimensions the reflection marker took was analyzed.

Results: Five healthy volunteers (Medium age 27 years, Female) who received written consent to the study were investigated. A spiral motion was observed in the trajectory of all reflection markers. A walking motion was observed in which the right and left sides of the body alternately swam to the back. Each reflex marker body site was moving headward as if contacting the floor with either the left or right side having 0 to the vertical Z-axis of gravity; the opposite left or right side walked with repeated movements floating from the floor and raising the $\mathbf{Z}$ coordinate. 3D Space + 1D time were observed in the human fundamental upward movement.

Conclusion: The Human Fundamental Upward Movement on the floor was observed Spiral Trajectory and the Walking of 3D+1D Space and Time.

Keywords: Human Fundamental Movement, Upward movement, Positioning Change, Transfer, Biomechanics

\section{INTRODUCTION}

Many studies of fundamental human movements have classically focused on a person standing upright in the direction of gravity. Old materials are left for the analysis of animals, children, men and women, the elderly, babies and dancers from standing to walking [1,2]. As a characteristic of human beings, interest in bipedal walking is very high, and there are many papers on the mechanism of walking $[3,4]$. Since biomechanical analysis machines cannot analyze the reflection markers because they are hidden from the camera, motion analysis such as standing and walking is particularly preferred, and the number of papers may be very large. There are few biomechanical studies on the movement of a person lying on a floor or bed in a position perpendicular to the direction of gravity. The recumbent position is categorized as a restricted posture for the motion analysis because of the difficulty to put markers on the back side.
The reflective markers tend to be hidden in complex motion. Hospitals and institutions provide an environment where many patients and older people spend most of their time in bed as a recuperation environment. The problems of pressure sores and joint contractors are still a global concern, affecting the health and quality of life of patients and families and the cost of national health care [5-10]. Basic biomechanics research can be an important source. In this study, the biomechanical analysis was performed, focusing on the upward movement in a supine position on the floor. Biomechanics is a method of objectively understanding human movement. In this study, a biomechanical analysis was performed focusing on the upward movement in a supine position on the floor.

\section{METHODS}

Healthy volunteers were recruited by a snowball sampling. After the researchers performed 3D spatial calibration, the subjects

Copyright: (C) 2021. Hiroko Tadaura. This is an open-access article distributed under the terms of the Creative Commons Attribution License, which permits unrestricted use, distribution, and reproduction in any medium, provided the original author and source are credited. 
were fitted with reflection markers using an 11 segments model. The subjects performed upward movements from lying position on the floor for three times each. The three procedures were analyzed using a 3D motion analysis MoveTr 3D (LibraryC) under the 4 CCD cameras. The motion traces were examined. The movement of the reflection marker to the $Z$ axis was analyzed with the vertical direction of the gravity as the $Z$ axis. It was observed from $X Y$ plane, $X Z$ plane, $Z Y$ plane, and how many dimensions the reflection marker took was analyzed.

\section{RESULTS}

Five female volunteers aged 30's who finished the training was analyzed in Spiral and Parallel movements for 5 times each.

As a result of observing the trajectory of upward movement from the supine resting position, Spiral Trajectory was observed
(Figure 1). All reflection markers were spiral locus.

Motion trace to $\mathrm{Z}$ axis (Head) was moving up and down (Figure 2).

Motion trace to $\mathrm{Z}$ axis (Shoulders) was moving up and down alternately (Figure 3). When either side of the floor was in contact with the floor, the opposite side was in the space, and when the side that was in the space was in contact with the floor, the opposite side floated in the space. Motion trace to $Z$ axis (Elbows, Iliac crest, Knees, Feet and Fingers) showed the same as Shoulders (Figure 4,5,6 and 7).

These movements were like Walking on the floor using each body parts (Figure 8). Checking the camera from each side of the three-dimensional space shows that these motions were three-dimensional (Figure 9).
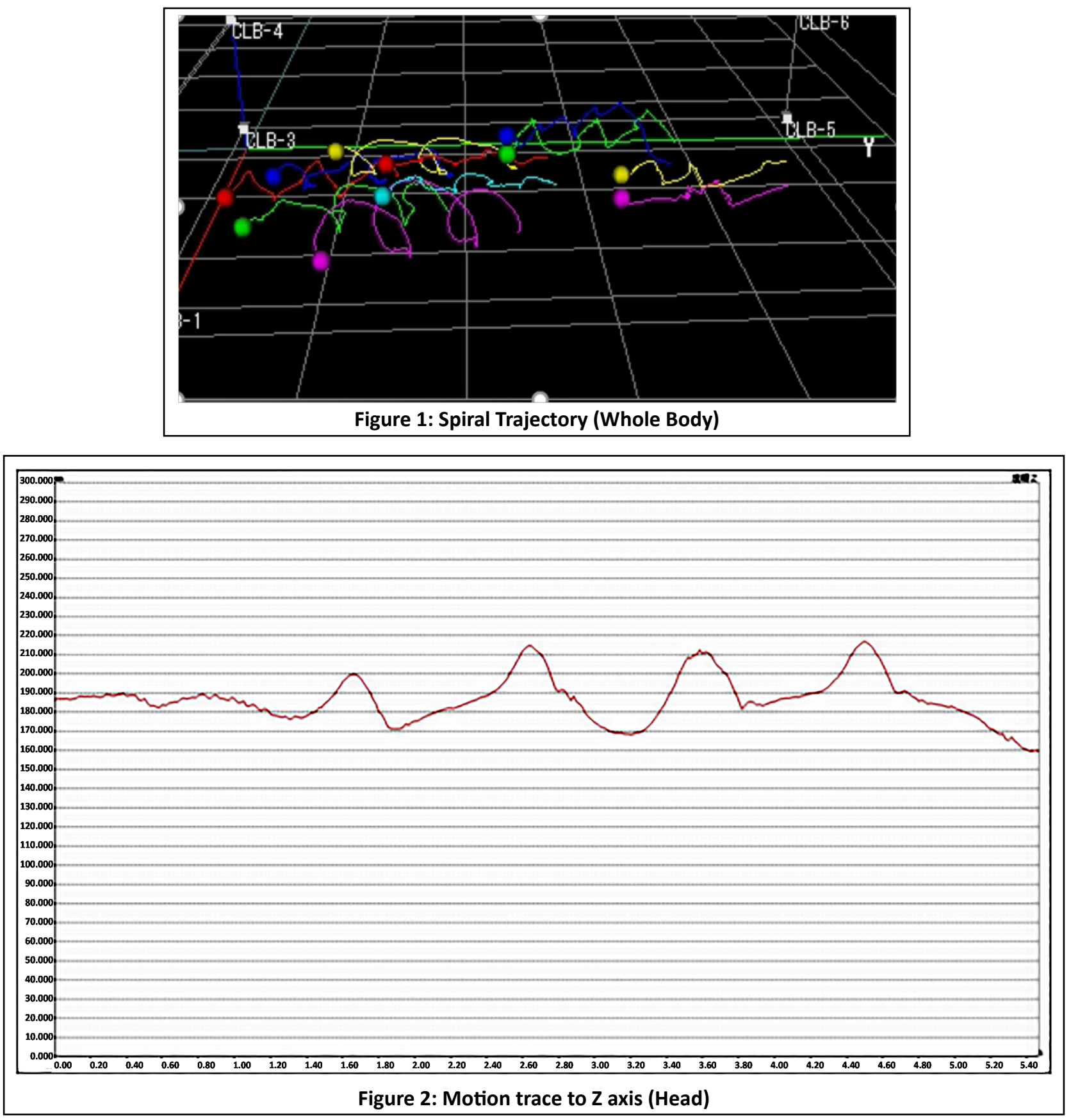

Citation: Hiroko Tadaura (2021). Human Fundamental Upward Movement; Spiral Trajectory and Walking of 3D + 1D Space and Time. POJ NursPrac Res. 5(1):1-7. DOI: https://doi.org/10.32648/2577-9516/5/1/3. 

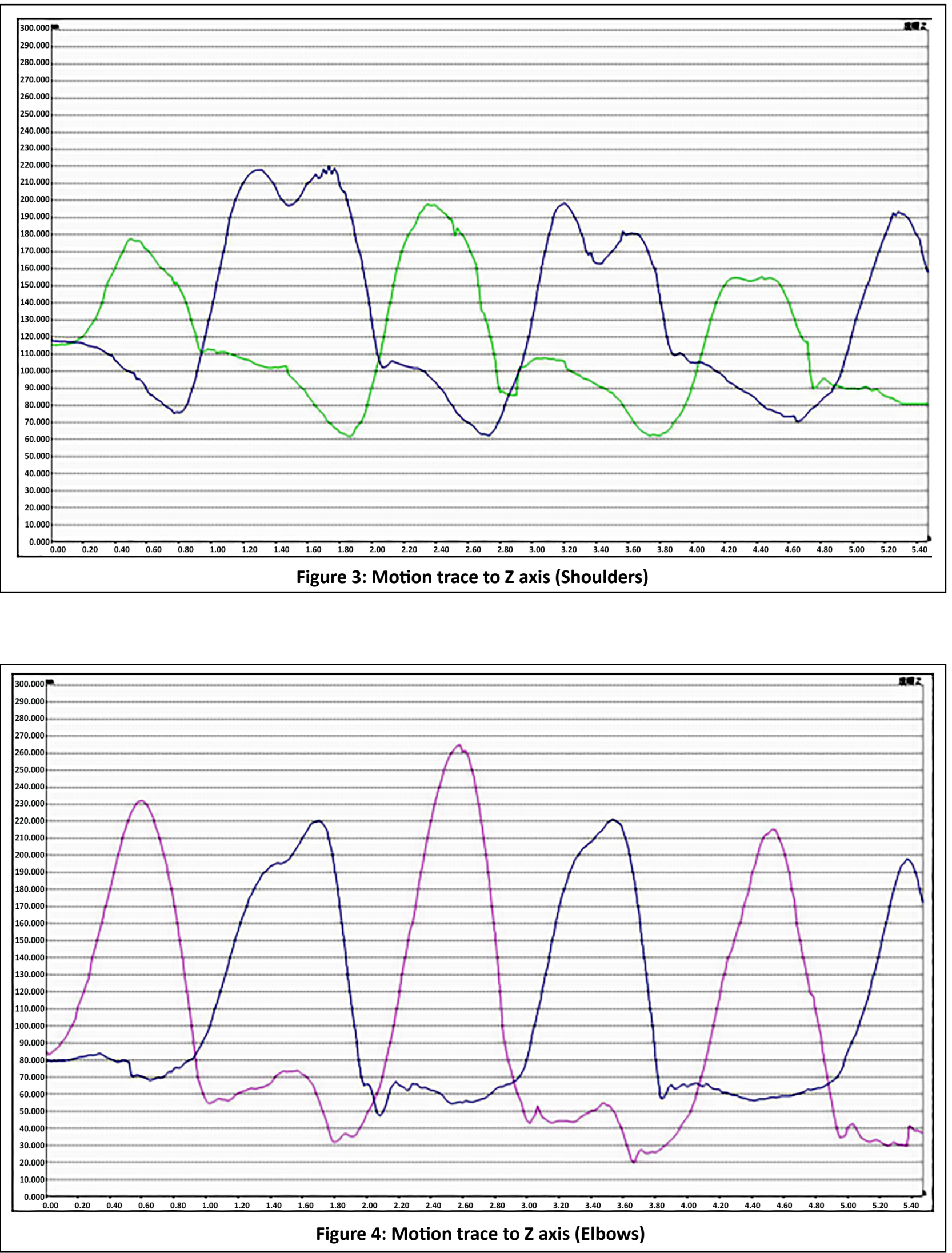

Citation: Hiroko Tadaura (2021). Human Fundamental Upward Movement; Spiral Trajectory and Walking of 3D + 1D Space and Time. POJ NursPrac Res. 5(1):1-7. DOI: https://doi.org/10.32648/2577-9516/5/1/3. 


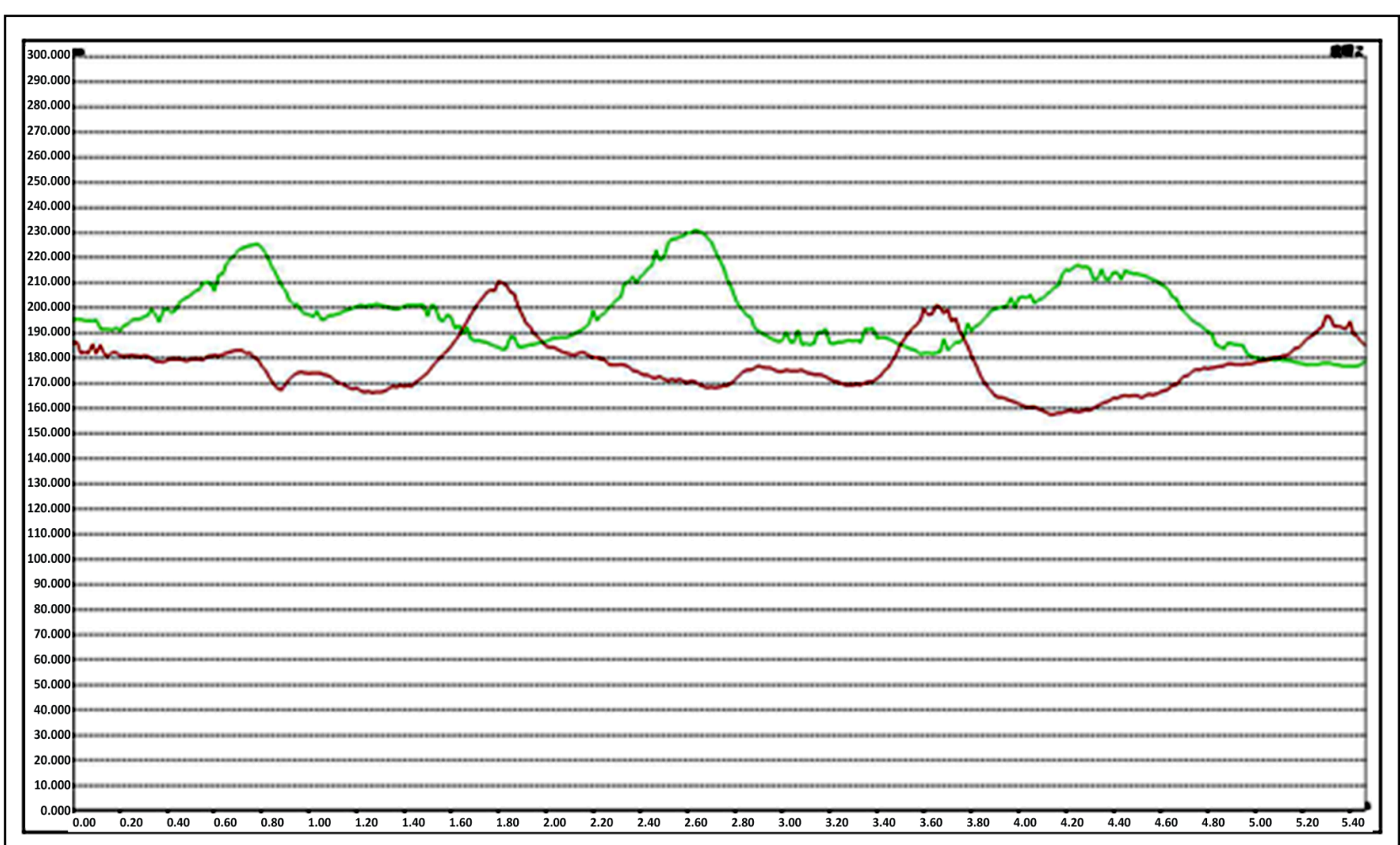

Figure 5: Motion trace to $\mathrm{Z}$ axis (Iliac crest)

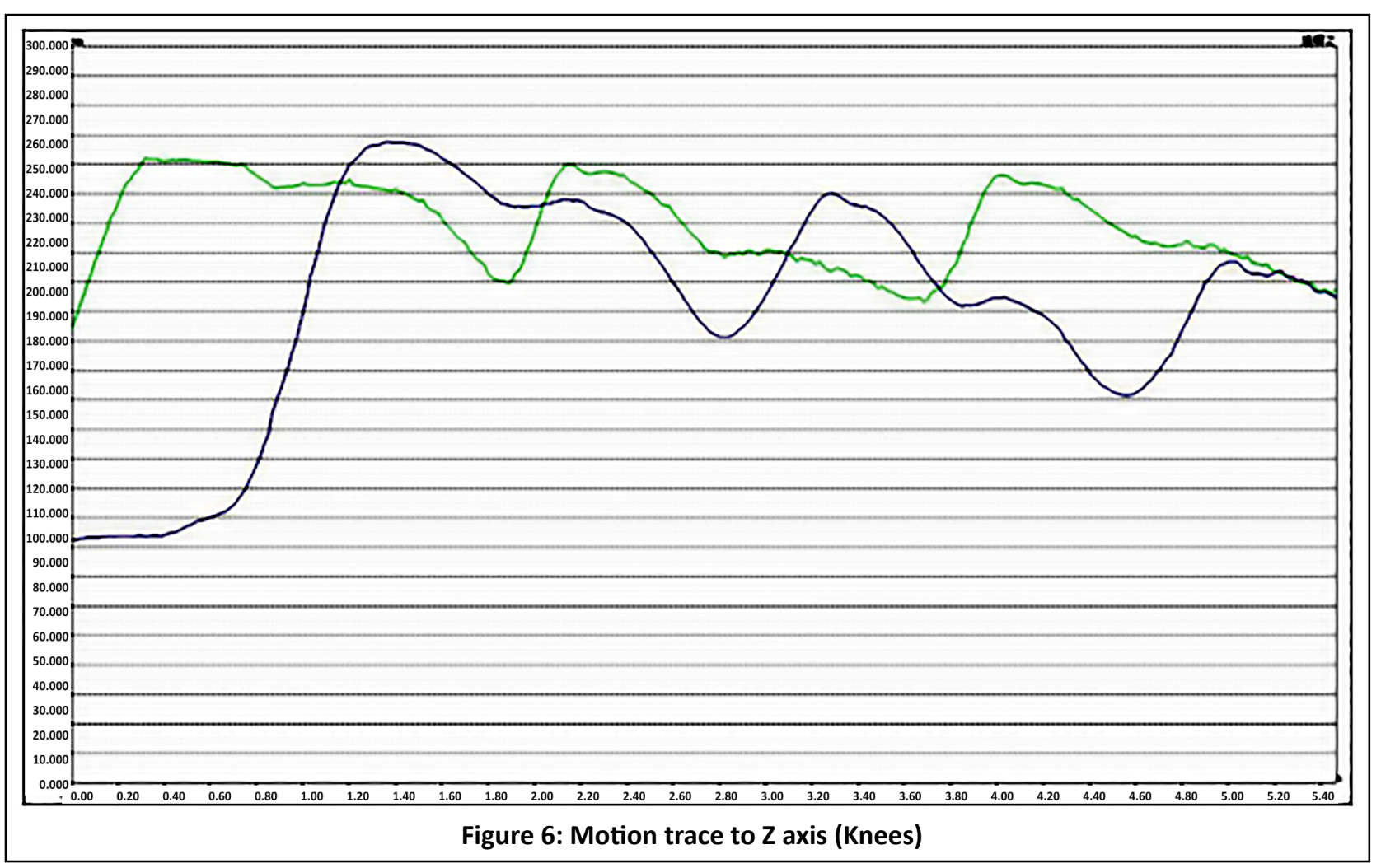

Citation: Hiroko Tadaura (2021). Human Fundamental Upward Movement; Spiral Trajectory and Walking of 3D + 1D Space and Time. POJ NursPrac Res. 5(1):1-7. DOI: https://doi.org/10.32648/2577-9516/5/1/3. 

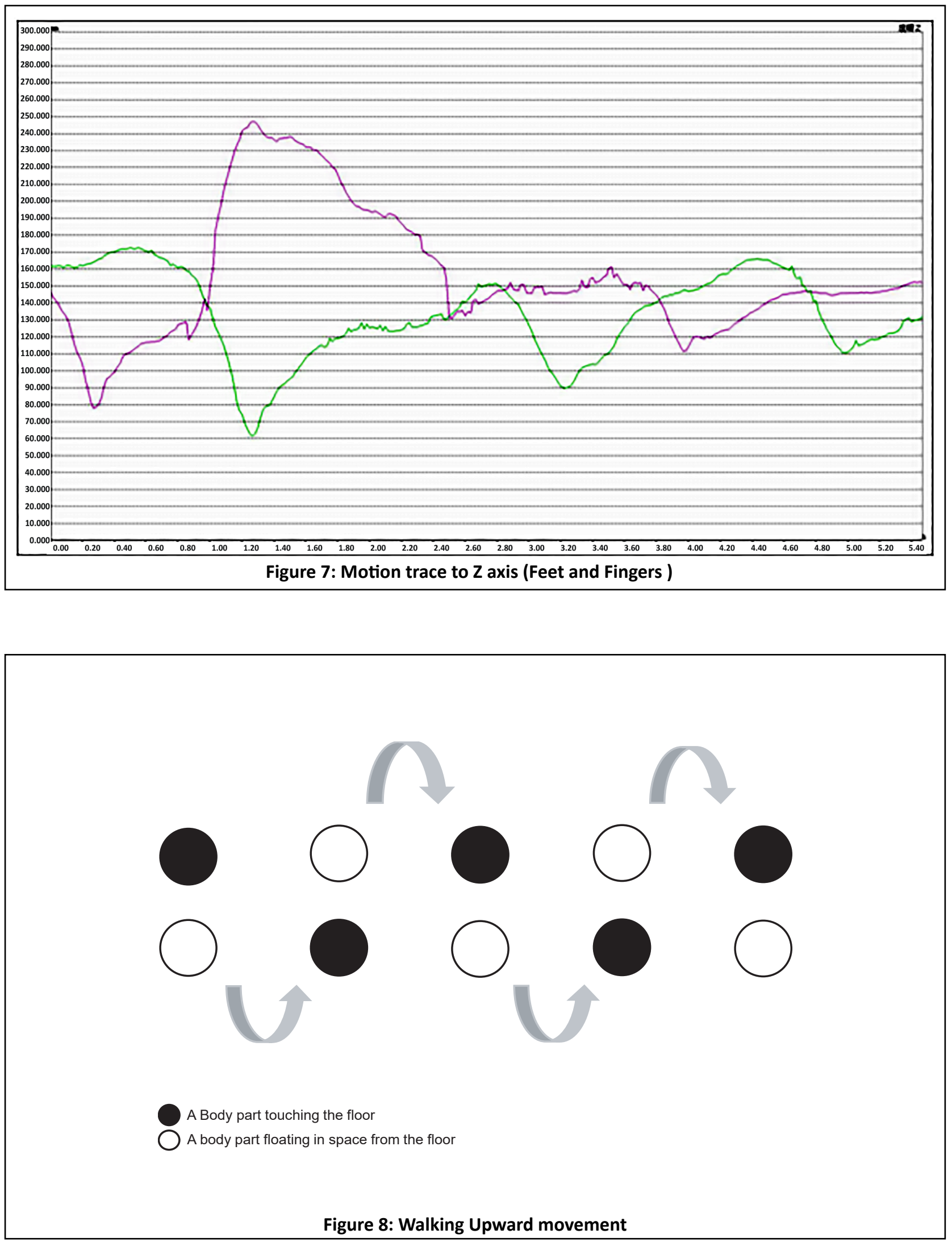

Citation: Hiroko Tadaura (2021). Human Fundamental Upward Movement; Spiral Trajectory and Walking of 3D + 1D Space and Time. POJ NursPrac Res. 5(1):1-7. DOI: https://doi.org/10.32648/2577-9516/5/1/3. 


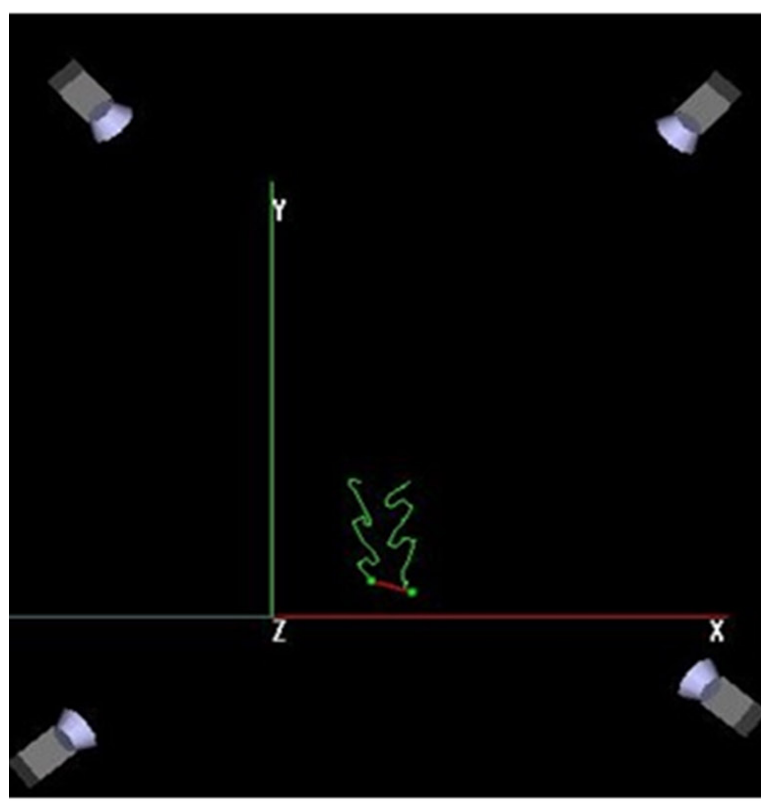

XZ PLANE

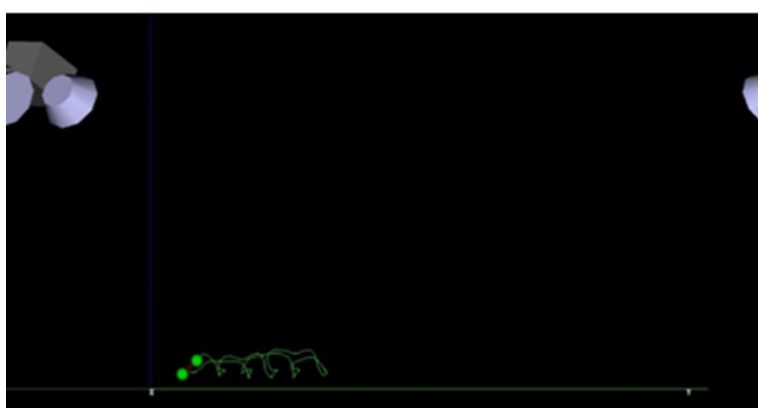

XY PLANE

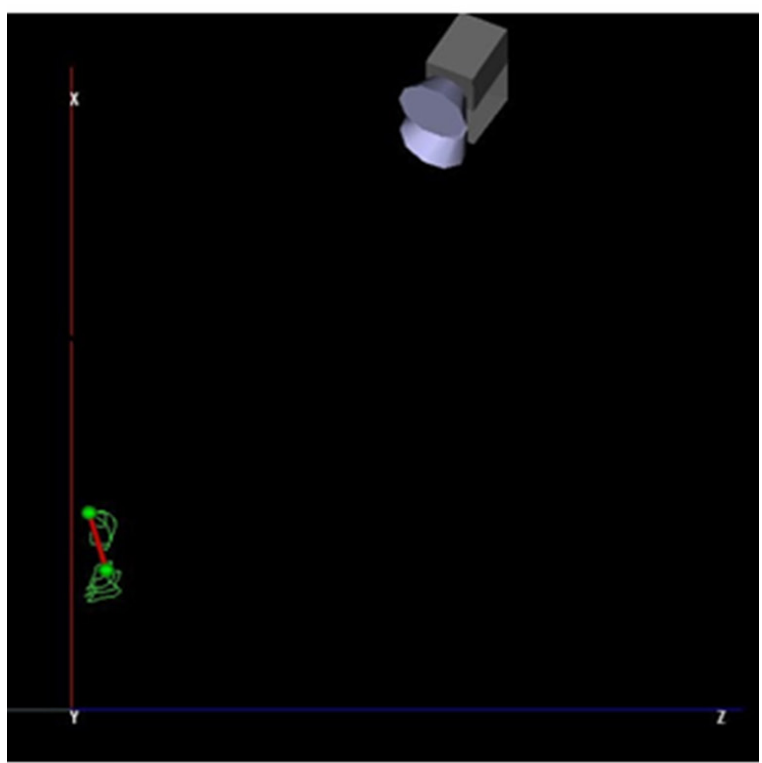

YZ PLANE

Figure 9: XZ plane, $X Y$ plane, and $Y Z$ plane

\section{DISCUSSION}

Previous studies on nursing textbooks have reported that upward movement is supported by pulling the patient up in a straight line with a sheet or the like [11-13]. Nurses hold their hands under the patient's body and pull the patient up in a straight line toward the head [11-13]. During this movement process, the patient physically and passively moved upward while remaining in a recumbent position, suggesting that the patient's state dimension may be 1-2 dimensions of the circle. However, biomechanics observed that when the upward movement of the human body was observed, everybody part showed a spiral movement trajectory, which was not linear. The study also showed that people move step by step like walking on the floor, rather than moving physically from a bed rest to a destination point with a big move. In the walking motion, the left or right side repeatedly moved to the destination while making a circle in the space while one on the right or left side touched the floor and supported the weight. The camera observed threedimensional motion, suggesting that $3 D+1 D$ space and time as well as the time dimension are upward motions.

Support for repositioning is usually provided for people who cannot move themselves. It has also been proven that the use frequency is high even in the nursing technology [14-21]. Many studies have shown that decubitus ulcers and limited range of motion are still major clinical problems [10,14-21]. The development of environment devices is important, but it is also necessary to reconsider the support for movement itself. This study is likely to serve as a reference. The spiral motion of each marker suggests that each joint is in a circular motion. Including natural and basic exercise support in daily care is likely to function as a range of motion preventer for contractions, pressure ulcers, and other disorders. If a healthy person who does not have pressure sores or joint contractures is constantly taking care of himself, it is necessary to observe the movements of a healthy person. Rather than applying special movements to patients, the elderly and the disabled, it may be possible to prevent them by basing them on the movements and characteristics of healthy people. If motion is related to the living dimension, applying the $3 D+1 D$ motion support observed in this study would place the patient's life state in the 3D + 1D dimension. Maintaining a higher level of life in the clinic may also contribute to health and longevity.

\section{CONCLUSION}

Biomechanical properties of the human fundamental upward movement of the floor in the supine position was Spiral Trajectory and the Walking of 3D+1D Space and Time.

\section{ETHICS APPROVAL}

The study was conducted with oral and written consent and ethical considerations based on the Declaration of Helsinki and the Medical Research Ethics Guidelines.

\section{COMPETING INTERESTS}

The author declares no competing interests. 


\section{FUNDING}

This work was supported by JSPS KAKENHI Grant Numbers JP17H01692, JP18689051.

\section{ACKNOWLEDGEMENTS}

We would like to express our gratitude to subjects and their cooperation in this study.

\section{References}

1. Eadweard Muybridge. Muybridge's Complete Human and Animal Locomotion: All 781 Plates from the 1887 Animal Locomotion: New Volume 2 (Reprint of original volumes 5-8) Hardcover - July 1, 1979.

2. Eadweard Muybridge. Animals in Motion (Dover Anatomy for Artists) Hardcover - Illustrated, June 1, 1957.

3. Bohannon WR, Andrews WA. Normal walking speed: a descriptive meta-analysis. Physiotherapy. 2011;97(3):182189.

4. Boyer AK, Johnson TR, Banks JJ. Systematic review and meta-analysis of gait mechanics in young and older adults. Exp Gerontol. 2017;95:63-70.

5. Michael J Clancy. Pressure redistribution devices: what works, at what cost and what's next?. J Tissue Viability. 2013;22(3):57-62.

6. Prevention and Treatment of Pressure Ulcers/Injuries: Clinical Practice Guideline. The International Guideline, 3rd Edition. 2019. Available from: http://www. internationalguideline.com/.

7. Medical Advisory Secretariat. Pressure ulcer prevention: an evidence-based analysis. Ont Health Technol Assess Ser. 2009;9(2):1-104.

8. Matthew D Redelings, Nolan E Lee, Frank Sorvillo. Pressure Ulcers: More Lethal Than We Thought?. Adv Skin Wound Care. 2005;18(7):367-72.

9. W M Bortz 2nd. The disuse syndrome. West J Med. 1984;141(5):691-4.

10. Ito S, Tadaura H. Trends and factors in nursing research in Japan on disuse syndrome, issues to be addressed. The 26th academic conference of the Japanese Society of Rehabilitation and Nursing. 2014; 57. (Japanese)

11. Tadaura H, Kunii K. Comparison of German, Japanese, and Chinese Basic Nursing Textbooks in Helping with Postural Change and Mobile Transfer. The 27th Academic Meeting of Japan Society for Rehabilitation Nursing, Westa kawagoe, Saitama, Japan, November,2015. (Japanese)

12. Tadaura H. New innovation of Nursing movement care. Nursing World Conference, Dubai, 27-29 October, 2016.

13. Tadaura H. Nursing Relativity -Prevention and Management of Pressure Ulcers. International Conference on Wound Care, Tissue Repair and Regenerative Medicine, London, UK, June 14-15, 2018.
14. Tadaura H, Tokunaga K. From Body mechanics to Kinaesthetik/Kinaesthetics. The Japanese Journal of Nursing Science. 2006;31(10):49-53. (Japanese)

15. Tadaura H. Guide of new movement Care, From Body mechanics to Kinaesthtics. VIVO. 2016;8:30-34. (Japanese)

16. Tadaura H. Movement support to prevent Pressure Ulcer using Kinaesthetik/Kinaesthetics: Actual care using Kinaesthetik/Kinaesthetics. The Japanese Journal of Nursing Science. 2016;31(3):15-39. (Japanese)

17. Tadaura H, Tokunaga K. Nursing Application of Kinaesthetik/ Kinaesthetics concept; Usefulness and evidence of Kinaesthetik/Kinaesthetics. EBNursing. 2005;5(4):534-539. (Japanese)

18. Tadaura H. Chapter of Kinaesthetics, Supervision Dai et al: The Theory of Home Nursing (2): Technology to Support, Home Care, Nursing Graphicus, Tokyo, Japan 2018. (Japanese)

19. Tadaura H, Tokunaga K. Chapter 2 Positioning and its historical consideration, Editorial supervision : Makiko T, Positioning - Basic and practice of Position management, Nakayama Shoten Co Ltd, 2013;37-44. (Japanese)

20. Tadaura H. Chapter 4 Care and evidence for Cancer patients, 2. Investigation of Comfort and Evidence from patients: Positioning, Editorial supervision: Asano M, Cancer Nursing, Nankodo. 2012;17(2):281-284. (Japanese)

21. Tadaura H, Kumiko Sakaki. Score of SOPMAS in the Kinaesthetics and Body Mechanics Groups. The 18th Annual Congress of Japanese Society of Pressure Ulcer, Yokohama, Japan, 2016. (Japanese) 